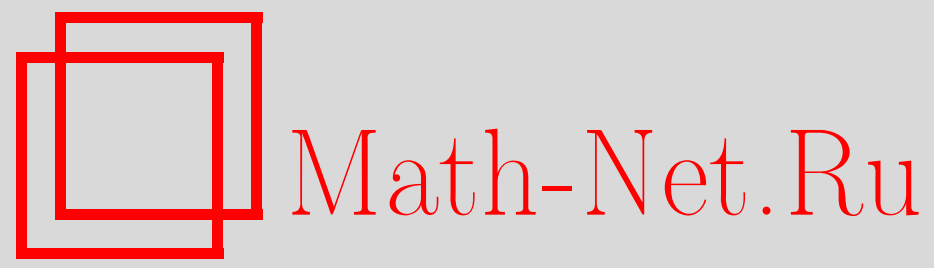

В. А. Копытцев, В. Г. Михайлов, Условия сходимости к распределению Пуассона для чисел решений случайных включений, Матем. вопр. криптогр., 2012, том 3 , выпуск 3, 35-55

DOI: https://doi.org/10.4213/mvk60

Использование Общероссийского математического портала Math-Net.Ru подразумевает, что вы прочитали и согласны с пользовательским соглашением

http: //www. mathnet.ru/rus/agreement

Параметры загрузки:

IP : 54.198 .55 .26

26 апреля 2023 г., 06:09:02 
УДК: 519.212.2+519.214.5

\title{
Условия сходимости \\ к распределению Пуассона \\ для чисел решений случайных включений
}

\author{
В. А. Копытцев ${ }^{1}$, В. Г. Михайлов ${ }^{2}$ \\ ${ }^{1}$ Академия криптографии Российской Федерации, Москва \\ ${ }^{2}$ Математический институт им. В. А. Стеклова РАН, Москва
}

Получено 20.V.2011

Пусть $F$ - случайное отображение $n$-мерного пространства $V^{n}$ над конечным полем $G F(q)$ в $T$-мерное пространство $V^{T}$ над тем же полем, и $D \subset V^{n}, B \subset V^{T}$. Выведены новые достаточные условия сходимости при $n, T \rightarrow \infty$ распределения числа решений системы включений $x \in D$, $F(x) \in B$ к распределению Пуассона.

Ключевые слова: случайные включения, системы случайных уравнений, число решений, предельная теорема Пуассона

Conditions of convergence to the Poisson distribution for the number of solutions of random inclusions

\author{
V. A. Kopytcev ${ }^{1}$, V. G. Mikhailov ${ }^{2}$ \\ ${ }^{1}$ Academy of Cryptography of the Russian Federation, Moscow \\ ${ }^{2}$ Steklov Mathematical Institute of RAS, Moscow
}

Abstract. Let $F$ be a random mapping of $n$-dimensional space $V^{n}$ over the finite field $G F(q)$ into $T$-dimensional space $V^{T}$ over the same field; let $D \subset V^{n}, B \subset V^{T}$. For the number of solutions of random inclusions $x \in D, F(x) \in B$ we find new sufficient conditions of weak convergence to the Poisson law as $n, T \rightarrow \infty$.

Key words: random inclusions, systems of random equations, number of solutions, Poisson convergence

Citation: Mathematical Aspects of Cryptography, 2012, vol. 3, no. 3, pp. 35-55 (Russian). 


\section{§1. Введение}

В работе исследуется распределение числа $\xi(D, F, B)$ решений системы включений

$$
x \in D, \quad F(x) \in B,
$$

где $F(x)=\left(F_{1}(x), \ldots, F_{T}(x)\right): V^{n} \rightarrow V^{T}$ - случайное отображение пространства $n$-мерных векторов $V^{n}$ над полем $K=G F(q)$ в пространство $V^{T}$, а $D$ и $B$ - некоторые множества $n$-мерных и $T$-мерных векторов над полем $K$.

Рассмотрим класс включений вида (1.1) с функцией

$$
F(x)=A_{1} x+A_{2} f(x)+S(x),
$$

где $A_{1}$ и $A_{2}-$ независимые случайные матрицы (над полем $K$ ) размеров $T \times n$ и $T \times m$ соответственно, $f(x)=\left(f_{1}(x), \ldots, f_{m}(x)\right)$ - заданное отображение, удовлетворяющее условию $f\left(0^{n}\right)=0^{m}$, а $S(x)=\left(S_{1}(x), \ldots, S_{T}(x)\right)$ - случайное отображение, не зависящее от матриц $A_{1}, A_{2}$. Случай, когда $S(x) \equiv 0^{T}$, был исследован ранее в работах $[1,2]$.

Нетрудно заметить, что класс включений с функцией $F(x)$ вида (1.2) c $S(x) \not \equiv 0^{T}$ содержит системы полиномиальных уравнений над полем $K=$ $G F(q)$ вида

$$
\sum_{\substack{d_{1}, \ldots, d_{n} \in\{0, \ldots, q-1\} \\ 1 \leq d_{1}+\ldots+d_{n} \leq g_{t}}} a_{d_{1} \ldots d_{n}}^{(t)} x_{1}^{d_{1}} \ldots x_{n}^{d_{n}}=b_{t}, \quad t=1, \ldots, T,
$$

где $a_{d_{1} \ldots d_{n}}^{(t)}-$ независимые в совокупности случайные величины, а параметры $g_{t}$ зависят от номеров уравнений и удовлетворяют условиям $2 \leq g_{t} \leq$ $n(q-1), t=1, \ldots, T$. Возможность исследования случайных систем из полиномиальных уравнений разных степеней является главным отличием нынешней постановки задачи от $[1,2]$.

Сформулируем результаты работы. Далее предполагаем, что от параметров $n, T$ зависят числа $m=m(n)$, множества $D=D(n), B=B(T)$, матрицы $A_{s}=A_{s}(n, T), s=1,2$, и отображения $f(x), S(x)$.

Всюду далее элементы случайной матрицы $A=\left(A_{1}, A_{2}\right)$ независимы в совокупности и

$$
\mathbf{P}\left\{a_{t, j}=k\right\}=\frac{1+\Delta_{t, j}(k)}{q}, \quad k \in K,
$$

где $\sum_{k \in K} \Delta_{t, j}(k)=0, t=1, \ldots, T, j=1, \ldots, n+m$. Пусть

$$
\Delta=\max _{t, j, k}\left|\Delta_{t, j}(k)\right|<1 .
$$


Обозначим через $N\left(k_{1}, k_{2}, k_{3}, c, D\right)$ число решений уравнения $k_{1} u^{1}+k_{2} u^{2}+k_{3} u^{3}=c$ относительно тройки векторов $\left(u^{1}, u^{2}, u^{3}\right) \in D^{3}$, где $k_{1}, k_{2}, k_{3} \in K \backslash\{0\}, c \in V^{n}$. Пусть

$$
N(D)=\max _{k_{1}, k_{2}, k_{3}, c} N\left(k_{1}, k_{2}, k_{3}, c, D\right), \quad \rho(D)=N(D) /|D|^{2} .
$$

Очевидно, что $\rho(D) \leq 1$. Так как уравнение $k_{1} u^{1}+k_{2} u^{2}+k_{3} u^{3}=c$ при $c \in D$ и $k_{1}=k_{2}=1, k_{3}=-1$ имеет решения $\left(u^{1}, u^{2}, u^{3}\right)=(c, u, u)$, то

$$
|D|^{-1} \leq \rho(D) \leq 1
$$

Ниже будет играть важную роль условие $\rho(D(n)) \rightarrow 0$ при $n \rightarrow \infty$ (заметим, что тогда $|D(n)| \rightarrow \infty)$.

Сначала обратимся к случаю, когда отображение $f(x) \equiv 0^{T}$, и исследуем предельное поведение числа решений системы

$$
x \in D, \quad A x+S(x) \in B,
$$

где $A-$ случайная матрица размера $T \times n$, а $S(x)$ - случайное отображение, не зависящее от матрицы $A$.

Векторы $x$ и $c x, c \neq 0$, будем называть подобными.

Теорема 1. Пусть $D \subseteq V^{n} \backslash\left\{0^{n}\right\}$, причем в этом множестве нет подобных векторов, $F(x)=A x+S(x)$ и выполнены условия $n, T \rightarrow \infty, T \Delta \rightarrow 0$, $|D| \rightarrow \infty$,

$$
\begin{gathered}
\rho(D) \rightarrow 0, \quad \frac{\ln |B|}{\ln \rho(D)} \rightarrow 0, \\
q^{-T}|D||B| \rightarrow \lambda, \quad 0 \leq \lambda<\infty .
\end{gathered}
$$

Тогда распределение случайной величины $\xi(D, F, B)$ сходится к распределению Пуассона с параметром $\lambda$.

Теперь перейдем к системам общего вида

$$
x \in D, \quad A_{1} x+A_{2} f(x)+S(x) \in B .
$$

Заменой переменных

$$
y=(x, f(x))=\left(y_{1}=x_{1}, \ldots, y_{n}=x_{n}, y_{n+1}=f_{1}(x), \ldots, y_{n+m}=f_{m}(x)\right)
$$

эта система сводится к системе

$$
y \in D_{f}, \quad A y+S^{*}(y) \in B,
$$


где $S^{*}(y)=S\left(y_{1}, \ldots, y_{n}\right)$

$$
D_{f}=\{(x, f(x)) \mid x \in D\} \subseteq V^{n+m},
$$

а матрица $A=\left(A_{1}, A_{2}\right)$ получена объединением столбцов матриц $A_{1}$ и $A_{2}$. Число решений системы (1.11) совпадает с числом решений системы (1.12). Используя этот факт, мы выведем из теоремы 1 следующее утверждение.

Обозначим через $D_{f}^{\prime}$ множество всех векторов из $D_{f}$, не имеющих в $D_{f}$ подобных себе векторов.

Теорема 2. Пусть $D \subseteq V^{n} \backslash\left\{0^{n}\right\}, F(x)=A_{1} x+A_{2} f(x)+S(x)$ и выполнены условия $n, T \rightarrow \infty, T \Delta \rightarrow 0,|D| \rightarrow \infty$,

$$
\begin{gathered}
\frac{\left|D_{f}^{\prime}\right|}{|D|} \rightarrow 1, \\
\rho\left(D_{f}\right) \rightarrow 0, \quad \frac{\ln |B|}{\ln \rho\left(D_{f}\right)} \rightarrow 0,
\end{gathered}
$$

а также условие (1.10). Тогда распределение случайной величины $\xi(D, F, B)$ сходится к распределению Пуассона с параметром $\lambda$.

ЗАмЕчАНИЕ 1. В работе [1] было показано (см. доказательства следствий 4 и 2 в [1]), что условие (1.13) выполнено, если $D=V^{n} \backslash\left\{0^{n}\right\}$, а для множества $Q(f)=\left\{f_{1}(x), \ldots, f_{m}(x)\right\}$ справедливо хотя бы одно из условий $Q(f) \supseteq Q_{n}^{(2)}$ и $Q(f) \supseteq Q_{n}^{\left(t_{1}\right)} \cup Q_{n}^{\left(t_{2}\right)}$, где

$$
Q_{n}^{(t)}=\bigcup_{\substack{d_{1}, \ldots, d_{n} \in\{0, \ldots, q-1\} \\ d_{1}+\ldots+d_{n}=t}}\left\{x_{1}^{d_{1}} \ldots x_{n}^{d_{n}}\right\}
$$

и $2<t_{1}<t_{2}$, НОД $\left(t_{1}-1, t_{2}-1\right)=1$.

Пусть мощность множества $D \subseteq V^{n} \backslash\left\{0^{n}\right\}$ при переходе к пределу сравнима с мощностью пространства $V^{n}$. В этом случае условие $T \Delta \rightarrow 0$, использованное в теоремах 1 и 2 , можно заменить менее ограничительным условием $\Delta \rightarrow 0$.

Теорема 3. Пусть $F(x)=A_{1} x+A_{2} f(x)+S(x), D \subseteq V^{n} \backslash\left\{0^{n}\right\}, q^{-n}|D| \geq$ $\geq c>0$ и выполнены условия теоремы 2 с заменой условия $T \Delta \rightarrow 0$ условием $\Delta \rightarrow 0$. Тогда распределение случайной величины $\xi(D, F, B)$ сходится $\kappa$ распределению Пуассона с параметром $\lambda$.

ЗАмечАнИЕ 2. Если $K=G F(2)$, то в $V^{n}$ нет подобных векторов. Поэтому $D_{f}^{\prime}=D_{f}$, а условие (1.13) в теоремах 2 и 3 можно опустить. 
Дальнейший порядок изложения материала в статье следующий. Теоремы 1-3 доказываются в параграфах 2-4. В параграфе 5 теоремы 2 и 3 используются для исследования асимптотических свойств распределения числа решений систем включений из специального класса, содержащего заведомо совместные системы. В параграфе 6 рассматривается случай $K=G F(2)$. Здесь для $\xi(D, F, B)$ доказывается предельная теорема Пуассона, условия которой допускают асимптотическое вырождение распределений элементов матрицы $A$. Она распространяет теорему 3 работы [1] на случай $S(x) \not \equiv 0^{T}$.

\section{§2. Доказательство теоремы 1}

Доказательство теоремы 1 проведем по схеме доказательства теоремы 6 в [3]. Мы рассматриваем случай, когда $F(x)=A x+S(x)$. Пусть $I\{E\}$ обозначает индикатор случайного события $E$, а $J=D \times B$. Тогда

$$
\xi(D, F, B)=\sum_{(x, b) \in J} I\{F(x)=b\}
$$

В отличие от доказательства теоремы 6 в [3], которая доказывалась с помощью «многомерной» версии теоремы Б. А. Севастьянова о предельном распределении Пуассона суммы зависимых индикаторов (см. [4]), мы воспользуемся традиционной («одномерной») версией этой теоремы (см. [5, 6]). Для ее применения потребуется ряд определений. Как и в [3], введем множества

$$
\begin{aligned}
D_{k, j}=\left\{\left(x^{1}, \ldots, x^{k}\right)\right. & \left.\in D^{k}: \operatorname{rank}\left(x^{1}, \ldots, x^{k}\right)=j\right\}, \\
D_{k} & =\bigcup_{j=1}^{k-1} D_{k, j} .
\end{aligned}
$$

Отметим, что

$$
\left|D_{k, j}\right| \leq C_{k}^{j} q^{j(k-j)}|D|^{j}
$$

Положим

$$
J_{k}=\left\{\left(\left(x^{1}, b^{1}\right), \ldots,\left(x^{k}, b^{k}\right)\right) \in J^{k}:\left(x^{\alpha}, b^{\alpha}\right) \neq\left(x^{\beta}, b^{\beta}\right)(\alpha \neq \beta)\right\} .
$$

Определим исключительные множества $I_{k} \subset J_{k}$ равенством

$$
I_{k}=\left\{\left(\left(x^{1}, b^{1}\right), \ldots,\left(x^{k}, b^{k}\right)\right) \in J_{k}:\left(x^{1}, \ldots, x^{k}\right) \in D_{k}\right\} .
$$


Для доказательства теоремы достаточно проверить выполнение условий (они отличаются от аналогичных условий в [3] лишь заменой $A x$ на $F(x)$ )

$$
\begin{aligned}
& \sum_{(x, b) \in J} \mathbf{P}\{F(x)=b\} \rightarrow \lambda, \\
& \max _{(x, b) \in J} \mathbf{P}\{F(x)=b\} \rightarrow 0,
\end{aligned}
$$

и при всех $k=2,3, \ldots$ (далее для краткости используем обозначение $v^{i}=$ $\left.=\left(x^{i}, b^{i}\right)\right)$

$$
\begin{gathered}
\max _{\left(v^{1}, \ldots, v^{k}\right) \in J_{k} \backslash I_{k}}\left|\frac{\mathbf{P}\left\{F\left(x^{1}\right)=b^{1}, \ldots, F\left(x^{k}\right)=b^{k}\right\}}{\mathbf{P}\left\{F\left(x^{1}\right)=b^{1}\right\} \cdot \ldots \cdot \mathbf{P}\left\{F\left(x^{k}\right)=b^{k}\right\}}-1\right| \rightarrow 0, \\
\sum_{\left(v^{1}, \ldots, v^{k}\right) \in I_{k}} \prod_{i=1}^{k} \mathbf{P}\left\{F\left(x^{j}\right)=b^{j}\right\} \rightarrow 0, \\
\sum_{\left(v^{1}, \ldots, v^{k}\right) \in I_{k}} \mathbf{P}\left\{F\left(x^{1}\right)=b^{1}, \ldots, F\left(x^{k}\right)=b^{k}\right\} \rightarrow 0 .
\end{gathered}
$$

Проверка аналогов условий (2.4)-(2.8) в [3] опиралась на следующее утверждение (лемма 1 в [3]).

Лемма 1. Пусть $\left(x^{1}, \ldots, x^{k}\right) \in D_{k, j}, b^{1}, \ldots, b^{k} \in V^{T}$ и выполнено условие (1.5). Тогда при всех $k=1,2, \ldots$

$$
\mathbf{P}\left\{A x^{1}=b^{1}, \ldots, A x^{k}=b^{k}\right\} \leq\left(\frac{1+\Delta}{q}\right)^{j T},
$$

если $j \leq k-1$, а если $j=k$, то

$$
\left(\frac{1-\Delta}{q}\right)^{k T} \leq \mathbf{P}\left\{A x^{1}=b^{1}, \ldots, A x^{k}=b^{k}\right\} \leq\left(\frac{1+\Delta}{q}\right)^{k T} .
$$

Мы будем использовать лемму 1а - следствие леммы 1 и независимости случайного отображения $S$ и случайной матрицы $A$.

Лемма 1а. Пусть $F(x)=A x+S(x)\left(\right.$ или $\left.F(x)=A_{1} x+A_{2} f(x)+S(x)\right)$, $\left(x^{1}, \ldots, x^{k}\right) \in D_{k, j}, b^{1}, \ldots, b^{k} \in V^{T}$ и выполнено условие (1.5). Тогда при всех $k=1,2, \ldots$.

$$
\mathbf{P}\left\{F\left(x^{1}\right)=b^{1}, \ldots, F\left(x^{k}\right)=b^{k}\right\} \leq\left(\frac{1+\Delta}{q}\right)^{j T}
$$


если $j \leq k-1$, а если $j=k$, то

$$
\left(\frac{1-\Delta}{q}\right)^{k T} \leq \mathbf{P}\left\{F\left(x^{1}\right)=b^{1}, \ldots, F\left(x^{k}\right)=b^{k}\right\} \leq\left(\frac{1+\Delta}{q}\right)^{k T} .
$$

Соотношения (2.4)- (2.7) можно доказать, повторяя соответствующие рассуждения работы [3] с заменой $A x$ на $F(x)$ и используя лемму 1а вместо леммы 1. Эти выкладки мы не приводим.

Проверка же условия (2.8) требует использования нового хода рассуждений и нового условия (см. (1.9)), поскольку из-за наличия слагаемого $S$ в отображении $F$ некоторые леммы, использованные при доказательстве аналога (2.8) в [3], не применимы.

Итак, проверим соотношение (2.8). Введем множества

$$
\bar{D}_{k, j}=\left\{\left(x^{1}, \ldots, x^{k}\right) \in D_{k, j}: x^{\alpha} \neq x^{\beta}(\alpha \neq \beta)\right\} .
$$

Заметим, что $\bar{D}_{k, 1}=\varnothing, k=2,3, \ldots$ Положим $\bar{D}_{k}=\bigcup_{j=2}^{k-1} \bar{D}_{k, j}$. Тогда

$$
I_{k} \subseteq\left\{\left(v^{1}, \ldots, v^{k}\right):\left(x^{1}, \ldots, x^{k}\right) \in \bar{D}_{k}, b^{1}, \ldots, b^{k} \in B\right\} .
$$

Так как $\bar{D}_{2}=\bar{D}_{2,1}=\varnothing$, то $I_{2}=\varnothing$. При $k \geq 3$, используя (2.14), получаем:

$$
\begin{gathered}
\sum_{\left(v^{1}, \ldots, v^{k}\right) \in I_{k}} \mathbf{P}\left\{F\left(x^{1}\right)=b^{1}, \ldots, F\left(x^{k}\right)=b^{k}\right\}= \\
\leq \sum_{j=2}^{k-1} \sum_{\left(x^{1}, \ldots, x^{k}\right) \in \bar{D}_{k, j}} \sum_{\left(b^{1}, \ldots, b^{k}\right) \in B^{k}} \mathbf{P}\left\{F\left(x^{1}\right)=b^{1}, \ldots, F\left(x^{k}\right)=b^{k}\right\} .
\end{gathered}
$$

К выражению в правой части (2.15) применим оценку (2.9). Получим

$$
\begin{gathered}
\sum_{\left(v^{1}, \ldots, v^{k}\right) \in I_{k}} \mathbf{P}\left\{F\left(x^{1}\right)=b^{1}, \ldots, F\left(x^{k}\right)=b^{k}\right\} \leq \\
\leq \sum_{j=2}^{k-1} \sum_{\left(x^{1}, \ldots, x^{k}\right) \in \bar{D}_{k, j}}|B|^{k}\left(\frac{1+\Delta}{q}\right)^{j T} \leq \\
\leq \sum_{j=2}^{k-1} \frac{\left|\bar{D}_{k, j}\right|}{|D|^{j}}|B|^{k-j} \sum_{j=2}^{k-1}\left(\frac{(1+\Delta)^{T}|D||B|}{q^{T}}\right)^{j} .
\end{gathered}
$$


Так как в $D$ нет подобных векторов, то из (2.14) следует равенство

$$
\bar{D}_{k, j}=\left\{\left(x^{1}, \ldots, x^{k}\right) \in D_{k, j}: x^{\alpha} \neq c x^{\beta}(c \in K \backslash\{0\}, \alpha \neq \beta)\right\} .
$$

В [3] (см. лемму 6 и текст после нее) было показано, что для множества, указанного в правой части (2.17), выполнено неравенство

$$
\left|\bar{D}_{k, j}\right| \cdot|D|^{-j} \leq q^{k(k-j)} \rho(D) .
$$

Из этой оценки, условий (1.9), (1.10) и $T \Delta \rightarrow \infty$ следует, что выражение в правой части цепочки неравенств (2.16) стремится к нулю. Значит, условие (2.8) тоже выполнено. Теорема 1 доказана.

\section{§3. Доказательство теоремы 2}

Теперь $F(x)=A_{1} x+A_{2} f(x)+S(x)$, а $\xi(D, F, B)$ - число решений системы (1.11). Как мы уже отмечали, это число совпадает с числом решений системы (1.12).

Обратившись к определению функции $\rho(D)$, нетрудно проверить, что из равенства $\left|D_{f}\right|=|D|$, условий $\rho\left(D_{f}\right) \rightarrow 0$ и $\left|D_{f}^{\prime}\right|=|D|(1+o(1))$ следует соотношение $\rho\left(D_{f}^{\prime}\right) \rightarrow 0$. Поэтому из теоремы 1 и условий теоремы 2 выводим, что распределение числа решений $\xi\left(D_{f}^{\prime}, A y+S^{*}(y), B\right)$ системы

$$
y \in D_{f}^{\prime}, \quad A y+S^{*}(y) \in B,
$$

сходится к распределению Пуассона с параметром $\lambda$.

Осталось показать, что $\mathbf{P}\left\{\xi\left(D_{f}^{\prime}, A y+S^{*}(y), B\right)=\xi(D, F, B)\right\} \rightarrow 1$. Учитывая, что по лемме 1а для любых $y \in V^{n+m} \backslash\left\{0^{n+m}\right\}$ и $b \in B$ выполнено неравенство $\mathbf{P}\left\{A y+S^{*}(y)=b\right\} \leq((1+\Delta) / q)^{T}$, a $\left|D_{f}\right|=|D|$, получаем

$$
\begin{gathered}
\mathbf{E} \xi(D, F, B)-\mathbf{E} \xi\left(D_{f}^{\prime}, A y+S^{*}(y), B\right)= \\
=\mathbf{E} \xi\left(D_{f}, A y+S^{*}(y), B\right)-\mathbf{E} \xi\left(D_{f}^{\prime}, A y+S^{*}(y), B\right) \leq \\
\leq q^{-T}|B||D|\left(1-\frac{\left|D_{f}^{\prime}\right|}{|D|}\right)(1+\Delta)^{T} .
\end{gathered}
$$

Согласно условиям (1.13), (1.14), $T \Delta \rightarrow 0$ правая часть в цепочке соотношений (3.2) стремится к нулю. Так как

$$
\mathbf{P}\left\{\xi(D, F, B) \geq \xi\left(D_{f}^{\prime}, A y+S^{*}(y), B\right)\right\}=1,
$$

то из (3.2) следует, что

$$
\mathbf{P}\left\{\xi\left(D_{f}^{\prime}, A y+S^{*}(y), B\right) \neq \xi(D, F, B)\right\} \rightarrow 0 .
$$

Теорема 2 доказана. 


\section{§4. Доказательство теоремы 3}

По условию $F(x)=A_{1} x+A_{2} f(x)+S(x)$. Пусть

$$
D^{\prime}=\left\{x \in D:(x, f(x)) \in D_{f}^{\prime}\right\},
$$

а $J$ и $J^{\prime}$ обозначают множества $D \times B$ и $D^{\prime} \times B$ соответственно. Тогда

$$
\xi(D, F, B)=\sum_{(x, b) \in J} I\{F(x)=b\}, \quad \xi\left(D^{\prime}, F, B\right)=\sum_{(x, b) \in J^{\prime}} I\{F(x)=b\} .
$$

Доказательство проведем по схеме доказательства теорем 1 и 2 с использованием некоторых вспомогательных результатов из работы [2].

Сначала покажем, что

$$
\mathbf{P}\left\{\xi\left(D^{\prime}, F, B\right)=\xi(D, F, B)\right\} \rightarrow 1 .
$$

Введем множества

$$
\begin{gathered}
D_{k, j}=\left\{\left(x^{1}, \ldots, x^{k}\right) \in(D)^{k}: \operatorname{rank}\left(\left(x^{1}, f\left(x^{1}\right)\right), \ldots,\left(x^{k}, f\left(x^{k}\right)\right)\right)=j\right\}, \\
D_{k, j}^{\prime}=D_{k, j} \cap\left\{\left(x^{1}, \ldots, x^{k}\right) \in\left(D^{\prime}\right)^{k}\right\} .
\end{gathered}
$$

Для величин $\left|D_{k, j}^{\prime}\right|$ выполнены оценки, аналогичные оценкам (2.2):

$$
\left|D_{k, j}^{\prime}\right| \leq\left|D_{k, j}\right| \leq \sum_{s=1}^{j} C_{k}^{s} q^{s(k-s)}|D|^{s}<\sum_{s=1}^{j} C_{k}^{s} q^{s(k-s)} q^{n s} .
$$

Положим

$$
D_{k}^{\prime}=\bigcup_{j=1}^{k-1} D_{k, j}^{\prime}
$$

Для $x^{1}, \ldots, x^{k} \in D, k \geq 2, \alpha=1, \ldots, k$, введем величины

$$
\eta^{\alpha}\left(x^{1}, \ldots, x^{k}\right)=\sum_{i=1}^{n} \eta_{i}^{\alpha}\left(x^{1}, \ldots, x^{k}\right)
$$

где

$$
\eta_{i}^{\alpha}\left(x^{1}, \ldots, x^{k}\right)= \begin{cases}1, & \text { если } x_{i}^{\alpha} \neq 0 \text { и } x_{i}^{\beta}=0 \text { при всех } \beta \in\{1, \ldots, k\} \backslash\{\alpha\}, \\ 0 & \text { в противном случае. }\end{cases}
$$

Для $k=1$ положим $\eta^{1}\left(x^{1}\right)=\left\|x^{1}\right\|$ (напомним, что $\|x\|-$ число ненулевых элементов вектора $x$ ). При $0 \leq l \leq n$ определим множества

$$
\begin{gathered}
D_{k, j}(l)=\left\{\left(x^{1}, \ldots, x^{k}\right) \in D_{k, j}: \exists s_{1}, \ldots, s_{j} \in\{1, \ldots, k\}, s_{\alpha} \neq s_{\beta}(\alpha \neq \beta),\right. \\
\left.\eta^{s}\left(x^{s_{1}}, \ldots, x^{s_{j}}\right) \geq l \forall s \in\left\{s_{1}, \ldots, s_{j}\right\}\right\} .
\end{gathered}
$$

2012. T. 3. № 3. C. 35-55 
Пусть

$$
D_{k, j}^{\prime}(l)=D_{k, j}(l) \cap D_{k, j}^{\prime}
$$

Положим

$$
J_{k}^{\prime}=\left\{\left(\left(x^{1}, b^{1}\right), \ldots,\left(x^{k}, b^{k}\right)\right) \in\left(J^{\prime}\right)^{k}:\left(x^{\alpha}, b^{\alpha}\right) \neq\left(x^{\beta}, b^{\beta}\right)(\alpha \neq \beta)\right\} .
$$

Сформулируем два вспомогательных утверждения.

Лемма 2. Пусть $\left(x^{1}, \ldots, x^{k}\right) \in D_{k, j}(l), 0 \leq l \leq n, b^{1}, \ldots, b^{k} \in V^{T}$, и выполнено условие (1.5). Тогда при всех $k=1,2, \ldots$

$$
\mathbf{P}\left\{F\left(x^{1}\right)=b^{1}, \ldots, F\left(x^{k}\right)=b^{k}\right\} \leq\left(\frac{1+\Delta^{l}}{q}\right)^{j T},
$$

если $j \leq k-1$, а если $j=k$, то

$$
\left(\frac{1-\Delta^{l}}{q}\right)^{k T} \leq \mathbf{P}\left\{F\left(x^{1}\right)=b^{1}, \ldots, F\left(x^{k}\right)=b^{k}\right\} \leq\left(\frac{1+\Delta^{l}}{q}\right)^{k T} .
$$

Доказательство. Согласно лемме 1 из [2] соотношения (4.4), (4.5) выполнены (при указанных выше условиях и при любых $b^{1}, \ldots, b^{k} \in V^{T}$ ), если $S(x) \equiv 0^{T}$ и отображение $F(x)$ задано равенством $F(x)=A_{1} x+A_{2} f(x)$. В силу независимости отображений $A_{1} x+A_{2} f(x)$ и $S(x)$ лемма 2 вытекает из леммы 1 работы [2].

Лемма 3. Существуует такая функиия $\varepsilon(n) \rightarrow 0, n \rightarrow \infty$, что при всех $j=1, \ldots, k$ выполнено неравенство

$$
\left|D_{k, j} \backslash D_{k, j}(\ln n)\right| \leq q^{j n} \exp \left\{n\left(\ln \left(1-\frac{q-1}{q^{j}}\right)+\varepsilon(n)\right)\right\} .
$$

Лемма 3 является следствием леммы 2 из [2], в которой оценка (4.6) была доказана для $D=V^{n} \backslash\left\{0^{n}\right\}$.

Из (4.1) следует, что

$$
\mathbf{E}\left(\xi(D, F, B)-\xi\left(D^{\prime}, F, B\right)\right)=\sum_{(x, b) \in J \backslash J^{1}} \mathbf{P}\{F(x)=b\} .
$$

Разобьем сумму в правой части на две суммы:

$$
\sum_{(x, b) \in J \backslash J^{\prime}} \mathbf{P}\{F(x)=b\}=\Sigma_{1}+\Sigma_{2},
$$


где

$$
\begin{aligned}
& \Sigma_{1}=\sum_{(x, b) \in J \backslash J^{\prime},\|x\| \geq \ln n} \mathbf{P}\{F(x)=b\}, \\
& \Sigma_{2}=\sum_{(x, b) \in J \backslash J^{\prime},\|x\|<\ln n} \mathbf{P}\{F(x)=b\} .
\end{aligned}
$$

Используя оценку (4.5) (где $k=1$ ), получаем

$$
\begin{aligned}
& \Sigma_{1} \leq\left|D \backslash D^{\prime}\right||B|\left(\frac{1+\Delta^{\ln n}}{q}\right)^{T}= \\
= & \left(1-\frac{\left|D^{\prime}\right|}{|D|}\right) \frac{|D||B|}{q^{T}}\left(1+\Delta^{\ln n}\right)^{T} .
\end{aligned}
$$

Из условий (1.10), (1.14), неравенств $q^{-n}|D| \geq c>0$ и $\rho\left(D_{f}\right) \geq q^{-n}$ (см. (1.7)) следует, что при $n \rightarrow \infty$ выполнена оценка

$$
T=n(1+o(1))
$$

а из оценки (4.10) и условия $\Delta \rightarrow 0$ следует, что

$$
T \Delta^{\ln n} \rightarrow 0
$$

Из (4.9), (1.13), (1.10) и соотношения (4.11) получаем, что $\Sigma_{1}=o(1)$.

Вместе с этим, используя теперь оценку (2.10) (где $k=1$ ) и соотношения (4.10), $\Delta \rightarrow 0$, получаем

$$
\begin{gathered}
\Sigma_{2} \leq \sum_{0 \leq i<\ln n} C_{n}^{i}(q-1)^{i} \cdot|B|\left(\frac{1+\Delta}{q}\right)^{T}= \\
=O\left((n(q-1))^{\ln n}|B|\right)\left(\frac{1+\Delta}{q}\right)^{T}= \\
=\exp \left\{-n \ln q+T \ln (1+\Delta)+O\left(\ln ^{2} n\right)\right\}= \\
=\exp \{-n \ln q(1+o(1))\}=o(1) .
\end{gathered}
$$

Так как $\mathbf{P}\left\{\xi(D, F, B) \geq \xi\left(D^{\prime}, F, B\right)\right\}=1$, то, подставив в (4.8) и (4.7) выведенные соотношения для $\Sigma_{1}$ и $\Sigma_{2}$, получим:

$$
\mathbf{P}\left\{\xi\left(D^{\prime}, F, B\right) \neq \xi(D, F, B)\right\} \leq \mathbf{E}\left(\xi(D, F, B)-\xi\left(D^{\prime}, F, B\right)\right) \rightarrow 0 .
$$

Итак, соотношение (4.2) доказано. 
Теперь докажем предельную теорему Пуассона для случайной величины $\xi\left(D^{\prime}, F, B\right)$ (см. (4.1)), что и завершит доказательство теоремы 3. Для этого опять воспользуемся теоремой Б. А. Севастьянова. Определим исключительные множества $I_{k}^{\prime} \subset J_{k}^{\prime}$ равенством

$$
I_{k}^{\prime}=\left\{\left(\left(x^{1}, b^{1}\right), \ldots,\left(x^{k}, b^{k}\right)\right) \in J_{k}^{\prime}:\left(x^{1}, \ldots, x^{k}\right) \in D_{k}^{\prime} \cup\left(D_{k, k}^{\prime} \backslash D_{k, k}^{\prime}(\ln n)\right)\right\} .
$$

Согласно этому определению в исключительное множество $I_{k}^{\prime}$ вошли все наборы $\left(v^{1}, \ldots, v^{k}\right) \in J_{k}^{\prime}$, для которых набор $\left(x^{1}, \ldots, x^{k}\right)$ имеет ранг $j<k$, а в случае полного ранга $j=k-$ те наборы, в которых хотя бы один из векторов $x^{1}, \ldots, x^{k}$ имеет относительно мало ненулевых элементов на тех местах, где остальные $k-1$ векторов имеют нули.

Отметим сразу, что

$$
I_{k}^{\prime} \subseteq\left\{\left(x^{1}, \ldots, x^{k}\right) \in D_{k}^{\prime} \cup\left(D_{k, k}^{\prime} \backslash D_{k, k}^{\prime}(\ln n)\right)\right\} \cap\left\{b^{1}, \ldots, b^{k} \in B\right\},
$$

a

$$
\left.D_{k}^{\prime} \cup\left(D_{k, k}^{\prime} \backslash D_{k, k}^{\prime}(\ln n)\right)=D_{k}^{\prime}(\ln n) \cup\left(D_{k}^{\prime} \backslash D_{k}^{\prime}(\ln n)\right) \cup\left(D_{k, k}^{\prime} \backslash D_{k, k}^{\prime}(\ln n)\right)\right) .
$$

Предельная теорема Пуассона для случайной величины $\xi\left(D^{\prime}, F, B\right)$ (см. (4.1)) будет доказана, если убедиться, что выполнены условия (2.4)(2.8) для рассматриваемых в этом разделе отображения $F$ и множеств $I_{k}^{\prime}$ ). Приступим к проверке этих условий.

Соотношение (2.4) следует из (1.10) и (4.12), а соотношение (2.5) - из оценки (2.10) и условия $\Delta \rightarrow 0$. Соотношение (2.6) вытекает из определения исключительных множеств и неравенств (4.5).

Проверим соотношение (2.7) при $k \geq 2$. Из (4.14) следует равенство

$$
\sum_{\left(v^{1}, \ldots, v^{k}\right) \in I_{k}^{\prime}} \prod_{i=1}^{k} \mathbf{P}\left\{F\left(x^{i}\right)=b^{i}\right\}=S_{1}+S_{2},
$$

где

$$
\begin{gathered}
S_{1}=\sum_{\substack{\left(v^{1}, \ldots, v^{k}\right) \in J_{k}^{\prime} \\
\left(x^{1}, \ldots, x^{k}\right) \in D_{k}^{\prime}(\ln n)}} \prod_{i=1}^{k} \mathbf{P}\left\{F\left(x^{i}\right)=b^{i}\right\}, \\
S_{2}=\sum_{\substack{\left(v^{1}, \ldots, v^{k}\right) \in J_{k}^{\prime} \\
\left(x^{1}, \ldots, x^{k}\right) \in\left(D_{k}^{\prime} \backslash D_{k}^{\prime}(\ln n)\right) \cup\left(D_{k, k}^{\prime}\right)}} \prod_{k, k}^{k} \mathbf{P}\left\{F\left(x^{i}\right)=b^{i}\right\} .
\end{gathered}
$$


Используя условие $q^{-n}|D| \geq c>0$ и оценки (4.3), (4.4) (при $k=1$ ), (4.13), получаем

$$
\begin{gathered}
S_{1} \leq|B|^{k} \sum_{j=1}^{k-1}\left|D_{k, j}^{\prime}\right|\left(\frac{1+\Delta^{\ln n}}{q}\right)^{k T} \leq \\
\leq\left(\frac{|D||B|}{q^{T}}\right)^{k}\left(1+\Delta^{\ln n}\right)^{k T} \frac{1}{|D|^{k}} \sum_{j=1}^{k-1} \sum_{s=1}^{j} C_{k}^{s} q^{s(n+k-s)} \leq \\
\leq\left(\frac{|D||B|}{q^{T}}\right)^{k}\left(1+\Delta^{\ln n}\right)^{k T} \frac{1}{\left(c q^{n}\right)^{k}} \sum_{j=1}^{k-1} \sum_{s=1}^{j} C_{k}^{s} q^{s(n+k-s)} .
\end{gathered}
$$

Поэтому из (1.10), (4.11) и соотношения

$$
\frac{1}{q^{n k}} \sum_{j=1}^{k-1} \sum_{s=1}^{j} C_{k}^{s} q^{s(n+k-s)} \rightarrow 0, \quad n \rightarrow \infty,
$$

следует, что $S_{1} \rightarrow 0$.

Аналогичным образом с помощью соотношений $D_{k, j}^{\prime} \backslash D_{k, j}^{\prime}(\ln n) \subseteq$ $D_{k, j} \backslash D_{k, j}(\ln n),(2.12)$ (в нем надо положить $k=1$ и взять $F(x)=A_{1} x+$ $\left.A_{2} f(x)+S(x)\right)$ и (4.6) получаем оценки

$$
\begin{gathered}
S_{2} \leq\left(\frac{|D||B|}{q^{T}}\right)^{k}(1+\Delta)^{k T} \frac{1}{|D|^{k}} \sum_{j=1}^{k}\left|D_{k, j} \backslash D_{k, j}(\ln n)\right| \leq \\
=\frac{1}{\left(c q^{n}\right)^{k}} \sum_{j=1}^{k} q^{n j} \exp \left\{n \ln \left(1-\frac{q-1}{q^{j}}\right)+k T \ln (1+\Delta)+\varepsilon(n)\right\}=o(1) .
\end{gathered}
$$

Подставив в (4.15) выведенные соотношения для $S_{1}$ и $S_{2}$, получим (2.7).

Проверим соотношение (2.8). Опять $k \geq 2$. Введем множества

$$
\begin{aligned}
\bar{D}_{k, j}^{\prime} & =\left\{\left(x^{1}, \ldots, x^{k}\right) \in D_{k, j}^{\prime}: x^{\alpha} \neq x^{\beta}(\alpha \neq \beta)\right\}, \\
\bar{D}_{k, j}^{\prime}(\ln n) & =\left\{\left(x^{1}, \ldots, x^{k}\right) \in D_{k, j}^{\prime}(\ln n): x^{\alpha} \neq x^{\beta}(\alpha \neq \beta)\right\} .
\end{aligned}
$$

Заметим, что $\bar{D}_{k, 1}^{\prime}=\varnothing, k=2,3, \ldots$, и положим

$$
\bar{D}_{k}^{\prime}=\bigcup_{j=2}^{k-1} \bar{D}_{k, j}^{\prime}, \quad \bar{D}_{k}^{\prime}(\ln n)=\bigcup_{j=2}^{k-1} \bar{D}_{k, j}^{\prime}(\ln n) .
$$

2012. T. 3. № 3. C. $35-55$ 
Так как $\bar{D}_{2}^{\prime}=\bar{D}_{2,1}^{\prime}=\varnothing$, то $I_{k}^{\prime}=\varnothing$. Далее полагаем $k \geq 3$.

Из (4.14) следует равенство

$$
\sum_{\left(v^{1}, \ldots, v^{k}\right) \in I_{k}^{\prime}} \mathbf{P}\left\{F\left(x^{1}\right)=b^{1}, \ldots, F\left(x^{k}\right)=b^{k}\right\}=\bar{S}_{1}+\bar{S}_{2},
$$

где

$$
\begin{gathered}
\bar{S}_{1}=\sum_{\substack{\left(v^{1}, \ldots, v^{k}\right) \in J_{k}^{\prime} \\
\left(x^{1}, \ldots, x^{k}\right) \in \bar{D}_{k}^{\prime}(\ln n)}} \mathbf{P}\left\{F\left(x^{1}\right)=b^{1}, \ldots, F\left(x^{k}\right)=b^{k}\right\}, \\
\bar{S}_{2}=\sum_{\substack{\left(v^{1}, \ldots, v^{k}\right) \in J_{k}^{\prime} \\
\left(x^{1}, \ldots, x^{k}\right) \in\left(\bar{D}_{k}^{\prime} \backslash \bar{D}_{k}^{\prime}(\ln n)\right) \cup\left(D_{k, k}^{\prime} \backslash D_{k, k}^{\prime}(\ln n)\right)}} \mathbf{P}\left\{F\left(x^{1}\right)=b^{1}, \ldots, F\left(x^{k}\right)=b^{k}\right\} .
\end{gathered}
$$

Применим оценки (4.4) (при $l=\ln n)$ к сумме $\bar{S}_{1}$. Получим

$$
\begin{aligned}
\bar{S}_{1} & =\sum_{j=2}^{k-1} \sum_{\left(x^{1}, \ldots, x^{k}\right) \in \bar{D}_{k, j}^{\prime}(\ln n)} \sum_{\left(b^{1}, \ldots, b^{k}\right) \in B^{k}} \mathbf{P}\left\{F\left(x^{1}\right)=b^{1}, \ldots, F\left(x^{k}\right)=b^{k}\right\} \leq \\
& \leq \sum_{j=2}^{k-1}\left|\bar{D}_{k, j}^{\prime} \| B\right|^{k}\left(\frac{1+\Delta^{\ln n}}{q}\right)^{j T} \leq|B|^{k} \sum_{j=2}^{k-1}\left|D_{k, j}^{*}\right|\left(\frac{1+\Delta^{\ln n}}{q}\right)^{j T},
\end{aligned}
$$

где мы воспользовались отсутствием подобных векторов в $D_{f}^{\prime}$ и вытекающими из этого свойства соотношениями $\bar{D}_{k, j}^{\prime} \subseteq D_{k, j}^{*}$,

$$
D_{k, j}^{*}=\left\{\left(x^{1}, \ldots, x^{k}\right) \in D_{k, j}:\left(x^{\alpha}, f\left(x^{\alpha}\right)\right) \neq\left(c x^{\beta}, c f\left(x^{\beta}\right)\right), c \in K, \alpha \neq \beta\right\} .
$$

Применив (2.18) (в данном случае в (2.18) в качестве $D$ рассматривается $D_{f}$ ), получаем $\left|D_{k, j}^{*}\right| \leq q^{k(k-j)} \rho\left(D_{f}\right)|D|^{j}$. Используя эту оценку, условия теоремы и (4.11), от (4.18) приходим к соотношениям

$$
\begin{gathered}
\bar{S}_{1} \leq \rho\left(D_{f}\right)|B|^{k} \sum_{j=2}^{k-1} q^{k(k-j)}|D|^{j}\left(\frac{1+\Delta^{\ln n}}{q}\right)^{j T}= \\
=\rho\left(D_{f}\right)|B|^{k} \sum_{j=2}^{k-1} q^{k(k-j)}\left(\frac{|D|\left(1+\Delta^{\ln n}\right)^{T}}{q^{T}}\right)^{j}=O\left(\rho\left(D_{f}\right)|B|^{k}\right) \rightarrow 0 .
\end{gathered}
$$


Аналогичным образом с помощью леммы 1 (см. (2.10)) и соотношений $\bar{D}_{k, j}^{\prime} \backslash \bar{D}_{k, j}^{\prime}(\ln n) \subseteq D_{k, j} \backslash D_{k, j}(\ln n)$ получаем

$$
\bar{S}_{2} \leq|B|^{k} \sum_{j=2}^{k}\left|D_{k, j} \backslash D_{k, j}(\ln n)\right|\left(\frac{(1+\Delta)^{T}}{q^{T}}\right)^{j} .
$$

Используя оценку $\ln |B|=O(n)$ (она вытекает из (1.7), (1.14) и условия $\left.q^{-n}|D| \geq c>0\right)$, (4.6) и (4.10), приходим к выводу, что $\bar{S}_{2}=o(1)$. Подставив в (4.17) выведенные соотношения для $\bar{S}_{1}$ и $\bar{S}_{2}$, получим (2.8).

Таким образом, сходимость распределения случайной величины $\xi\left(D^{\prime}, F, B\right)$ к распределению Пуассона с параметром $\lambda$ доказана. Значит, доказана и теорема 3.

\section{§ 5. Класс включений, содержащий заведомо совместные системы}

В этом параграфе рассматривается распределение случайной величины $\xi\left(D, F, B \mid x^{0}\right), x^{0} \in V^{n}$, равной количеству решений системы включений

$$
x \in D, \quad F(x) \in F\left(x^{0}\right)+B .
$$

В случае, когда $D=V^{n}$ и $B=\left\{0^{T}\right\}$, система включений (5.1) с $F(x)=\left(F_{1}(x), \ldots, F_{T}(x)\right)$ представляет собой заведомо совместную систему уравнений

$$
F_{j}(x)=F_{j}\left(x^{0}\right), \quad j=1, \ldots, T,
$$

относительно $x \in V^{n}$.

Заменой переменных $u=x-x^{0}$ включения (5.1) приводятся к виду (1.1):

$$
u \in D-x^{0}, \quad F^{\left(x^{0}\right)}(u) \in B,
$$

где $F^{\left(x^{0}\right)}(u)=F\left(u+x^{0}\right)-F\left(x^{0}\right)$. Поэтому между случайными величинами $\xi\left(D, F, B \mid x^{0}\right)$ и $\xi(D, F, B)$ существует связь, выраженная равенствами

$$
\xi\left(D, F, B \mid x^{0}\right)=\xi\left(D-x^{0}, F^{\left(x^{0}\right)}, B\right) .
$$

При этом $\xi\left(D, F, B \mid 0^{n}\right)=\xi(D, F, B)$, если $F\left(0^{n}\right)=0^{T}$.

Далее $F(x)=A_{1} x+A_{2} f(x)+S(x)$, где $A_{1}$ и $A_{2}-$ случайные матрицы (над полем $K$ ) размеров $T \times n$ и $T \times m$ соответственно, $f(x)=$ $=\left(f_{1}(x), \ldots, f_{m}(x)\right)$ - заданное отображение, удовлетворяющее условию 
$f\left(0^{n}\right)=0^{m}$, а $S(x)=\left(S_{1}(x), \ldots, S_{T}(x)\right)-$ случайное отображение, не зависящее от матриц $A_{1}, A_{2}$. Пусть матрица $A=\left(A_{1}, A_{2}\right)$ размера $T \times(n+m)$ получена объединением матриц $A_{1}, A_{2}$; далее считается, что $\Delta=\Delta(A)$ удовлетворяет условию (1.5).

Определим отображение $f^{\left(x^{0}\right)}: V^{n} \rightarrow V^{m}$ равенством $f^{\left(x^{0}\right)}(x)=f(x+$ $\left.x^{0}\right)-f\left(x^{0}\right), x \in V^{n}$, и множество

$$
\left(D-x^{0}\right)_{f^{\left(x^{0}\right)}}=\left\{\left(x, f^{\left(x^{0}\right)}(x)\right) \mid x \in D-x^{0}\right\} \subseteq V^{n+m} .
$$

Заметим, что $\left|\left(D-x^{0}\right)_{f^{\left(x^{0}\right)}}\right|=|D|$. Построим множество $\left(D-x^{0}\right)_{f^{\left(x^{0}\right)}}^{\prime} \subseteq(D-$ $\left.x^{0}\right)_{f\left(x^{0}\right)}$ по такому же правилу, по какому для теоремы 2 из множества $D_{f}$ строилось множество $D_{f}^{\prime}$. Будем предполагать, что вектор $x^{0}=x^{0}(n)$ зависит от параметра $n$.

Теорема 2a. Пусть $D \subseteq V^{n} \backslash\left\{x^{0}\right\}, F(x)=A_{1} x+A_{2} f(x)+S(x)$ и выполнены условия $n, T \rightarrow \infty,|D| \rightarrow \infty, T \Delta \rightarrow 0$, (1.10),

$$
\begin{gathered}
\frac{\left|\left(D-x^{0}\right)_{f\left(x^{0}\right)}^{\prime}\right|}{|D|} \rightarrow 1, \\
\rho\left(\left(D-x^{0}\right)_{f^{\left(x^{0}\right)}}\right) \rightarrow 0, \quad \frac{\ln |B|}{\ln \rho\left(\left(D-x^{0}\right)_{f\left(x^{0}\right)}\right)} \rightarrow 0 .
\end{gathered}
$$

Тогда распределение случайной величины $\xi\left(D, F, B \mid x^{0}\right)$ сходится к распределению Пуассона с параметром $\lambda$.

Теорема 2а следует из теоремы 2, равенств (5.3) и

$$
F^{\left(x^{0}\right)}(x)=F\left(x+x^{0}\right)-F\left(x^{0}\right)=A_{1} x+A_{2} f^{\left(x^{0}\right)}(x)+S^{\left(x^{0}\right)}(x),
$$

где $S^{\left(x^{0}\right)}(x)=S\left(x+x^{0}\right)-S\left(x^{0}\right)$.

С помощью равенств (5.3) и (5.6) из теоремы 3 получается теорема $3 \mathrm{a}$.

Теорема 3a. Пусть $F(x)=A_{1} x+A_{2} f(x)+S(x), D \subseteq V^{n} \backslash\left\{x^{0}\right\}$, $q^{-n}|D| \geq c>0$ и выполнены условия теоремь $2 a$ с заменой условия $T \Delta \rightarrow 0$ условием $\Delta \rightarrow 0$. Тогда распределение случайной величины $\xi\left(D, F, B \mid x^{0}\right)$ сходится к распределению Пуассона с параметром $\lambda$. 
Сразу приведем условия для множества координатных функций $\left\{f_{1}(x), \ldots, f_{m}(x)\right\}$ отображения $f$, которые влекут условие $\rho\left(\left(D-x^{0}\right)_{f\left(x^{0}\right)}\right) \rightarrow$ $\rightarrow 0$ теорем 2а и 3а при $D=V^{n} \backslash\left\{x^{0}\right\}$. Пусть $\|z\|-$ число ненулевых элементов вектора $z$. Рассмотрим набор $d^{s}=\left(d_{1}^{s}, \ldots, d_{n}^{s}\right) \in\{0,1\}^{n}, s=1, \ldots, l$, где

$$
\begin{gathered}
\left\|d^{s}\right\| \geq 2, \quad s=1, \ldots, l, \\
\left\|\left(d_{1}^{s_{1}} d_{1}^{s_{2}}, \ldots, d_{n}^{s_{1}} d_{n}^{s_{2}}\right)\right\|=0, \quad 1 \leq s_{1}<s_{2} \leq l,
\end{gathered}
$$

и введем при $l=2,3, \ldots$ множество функций

$$
Q_{n}\left(d^{1}, \ldots, d^{l}\right)=\bigcup_{s=1}^{l}\left\{x_{1}^{d_{1}^{s}} \ldots x_{n}^{d_{n}^{s}}\right\} .
$$

Отметим, что множество $Q_{n}\left(d^{1}, \ldots, d^{l}\right)$ состоит из мономов $\varphi_{s}(x)=$ $=x_{1}^{d_{1}^{s}} \ldots x_{n}^{d_{n}^{s}}, s=1, \ldots, l$, содержащих только первые степени переменных. Согласно условию (5.7), степени самих мономов $\varphi_{s}(x)$ больше единицы, а условие (5.8) означает, что множества существенных переменных мономов из набора $Q_{n}\left(d^{1}, \ldots, d^{l}\right)$ попарно не пересекаются.

Условия, при которых $\rho\left(\left(D-x^{0}\right)_{f^{\left(x^{0}\right)}}\right) \rightarrow 0$, дает следующее утверждение, вытекающее непосредственно из оценки (3.10) в [1], полученной там при доказательстве теоремы 2.

Tеорема А. Пусть $\left\{f_{1}(x), \ldots, f_{m}(x)\right\} \supseteq Q_{n}\left(d^{1}, \ldots, d^{l}\right), l \leq m$. Тогдa при $D=V^{n} \backslash\left\{x^{0}\right\}$ для всех $x^{0} \in V^{n}$

$$
\rho\left(\left(D-x^{0}\right)_{f^{\left(x^{0}\right)}}\right) \leq 4 \prod_{s=1}^{l}\left(1-q^{-2\left\|d_{s}\right\|}\right) .
$$

Эта теорема позволяет нам доказать следующее утверждение. Рассмотрим множество функций (см. замечание 1)

$$
Q_{n}^{(2)}=\bigcup_{\substack{d_{1}, \ldots, d_{n} \in\{0, \ldots, q-1\} \\ d_{1}+\ldots+d_{n}=2}}\left\{x_{1}^{d_{1}} \ldots x_{n}^{d_{n}}\right\},
$$

которое состоит из всех мономов степени 2. 
Следствие 1. Пусть $\left\{f_{1}(x), \ldots, f_{m}(x)\right\} \supseteq Q_{n}^{(2)}, S\left(0^{n}\right)=0^{T}$, выполнены условия $n, T \rightarrow \infty$, (1.10), $\Delta \rightarrow 0$,

$$
|B| \leq q^{\delta T}, \quad \delta=\delta(n)=o(1) .
$$

Тогда распределение случайной величины $\xi\left(V^{n} \backslash\left\{x^{0}\right\}, F, B \mid x^{0}\right)$ (и в том числе распределение величины $\left.\xi\left(V^{n} \backslash\left\{0^{n}\right\}, F, B\right)\right)$ сходится к распределению Пуассона с параметром $\lambda$ равномерно относительно векторов $x^{0} \in V^{n}$.

ЗАМЕчАНИЕ 3. Класс случайных включений $F(x) \in F\left(x^{0}\right)+B$, где $F(x)=A_{1} x+A_{2} f(x)+S(x)$ и множество $\left\{f_{1}(x), \ldots, f_{m}(x)\right\}$ удовлетворяет условию теоремы А, содержит «заведомо совместные» системы уравнений, в левых частях которых стоят полиномы степеней $g_{t}$, где $2 \leq g_{t} \leq$ $\leq n(q-1), t=1, \ldots, T$ (см. (1.3)). Предельное распределение числа решений таких систем в случае, когда $K=G F(2)$, исследовалось в работе [7]. Предельная теорема Пуассона доказана в [7] при более слабых, чем у нас, условиях для параметра $\Delta$, но при дополнительных ограничениях на вес $\left\|x^{0}\right\|$ вектора $x^{0}$ (не допускаются векторы, имеющие при $n \rightarrow \infty$ «малый» вес, в частности, не допускается вектор $x^{0}=0^{n}$ ). В связи с этим отметим, что в следствии 1 сходимость к предельному распределению выполняется равномерно относительно всех векторов $x^{0} \in V^{n}$ (и в предположении, что $K-$ произвольное конечное поле). Для числа решений системы уравнений (1.3) эта сходимость равномерна и относительно величин $2 \leq g_{t} \leq n(q-1), t=1, \ldots, T$.

Доказательство следствия 1. Проверим, что условия (5.4) и (5.5) теоремы 2а выполнены равномерно по $x^{0} \in V^{n}$. Положим $\varphi_{i, j}(x)=x_{i} x_{j}$, где $1 \leq i<j \leq n$. Тогда

$$
\varphi_{i, j}^{\left(x^{0}\right)}(x)=\varphi_{i, j}\left(x+x^{0}\right)-\varphi_{i, j}\left(x^{0}\right)=x_{i} x_{j}+x_{j}^{0} x_{i}+x_{i}^{0} x_{j} .
$$

Пусть $x_{i}, x_{j} \in K \backslash\{0\}$ и $k \in K \backslash\{0\}$. При этом условии равенство $\varphi_{i, j}^{\left(x^{0}\right)}(k x)=$ $k \varphi_{i, j}^{\left(x^{0}\right)}(x)$ выполнено только в том случае, когда $k$ - единица поля $K$. Из условия $\left\{f_{1}(x), \ldots, f_{m}(x)\right\} \supseteq Q_{n}^{(2)}$ следует, что множество $\left\{f_{1}(x), \ldots, f_{m}(x)\right\}$ содержит все функции $\varphi_{i, j}(x), 1 \leq i<j \leq n$. Значит, $D_{f^{(x)}}^{\prime} \supseteq\left\{x \in V^{n}:\|x\| \geq 2\right\}$ при всех $x^{0} \in V^{n}$. Следовательно, при $n \rightarrow \infty$ соотношение (5.4) выполнено равномерно относительно векторов $x^{0} \in V^{n}$.

Проверим соотношения (5.5). Выберем в качестве $Q\left(d^{1}, \ldots, d^{l}\right)$ (см. (5.7)) множество функций $\varphi_{s}(x)=x_{2 s-1} x_{2 s}, s=1, \ldots, l$, где $l=[n / 2]-$ 
целая часть числа $n / 2$. Для такого множества

$$
\prod_{s=1}^{l}\left(1-q^{-2\left\|d_{s}\right\|}\right)=\left(1-q^{-4}\right)^{[n / 2]} \rightarrow 0
$$

при $n \rightarrow \infty$, и по теореме А выполнено первое из соотношений (5.5) (равномерно относительно $\left.x^{0} \in V^{n}\right)$. Кроме этого, отсюда и из теоремы А получаем, что

$$
\left|\ln \left(\rho\left(\left(D-x^{0}\right)_{f^{\left(x^{0}\right)}}\right)\right)\right| \geq-\ln \left(4\left(1-q^{-4}\right)^{[n / 2]}\right) \geq c n
$$

при некотором $c>0$. В свою очередь из условий (1.10) и (5.10) следует, что

$$
\ln |B|=o(T), \quad T=n(1+o(1)) .
$$

Из (5.11) и (5.12) вытекает, что второе соотношение в (5.5) выполнено равномерно относительно векторов $x^{0} \in V^{n}$. Таким образом, следствие 1 доказано.

\section{§6. Случай $K=G F(2)$}

Пусть

$$
\bar{Q}_{n}^{(g)}=\bigcup_{t=2}^{g} \bigcup_{\substack{d_{1}, \ldots, d_{n} \in\{0,1\} \\ d_{1}+\ldots+d_{n}=t}}\left\{x_{1}^{d_{1}} \ldots x_{n}^{d_{n}}\right\} .
$$

Для натуральных чисел $r$ и $g$ положим

$$
l(r, g)=\sum_{i=1}^{\min (r, g)} C_{r}^{i}
$$

Далее предполагаем, что числа $r=r(n), g=g(n)$ зависят от параметра $n$.

Теорема 4. Пусть $K=G F(2), F(x)=A_{1} x+A_{2} f(x)+S(x), D \subseteq$ $\subseteq V^{n} \backslash\left\{0^{n}\right\},\left\{f_{1}(x), \ldots, f_{m}(x)\right\} \supseteq \bar{Q}_{n}^{(g)}$ и выполнены условия $n, T \rightarrow \infty, r, g \rightarrow$ $\infty,|D| \rightarrow \infty, \rho\left(D_{f}\right) \rightarrow 0$,

$$
\begin{gathered}
\frac{\ln |B|}{\ln \rho\left(D_{f}\right)} \rightarrow 0, \\
\frac{2^{-T}|D||B| \rightarrow \lambda, \quad 0<\lambda<\infty,}{(1+\Delta)^{T}|\{x \in D:|| x||<r\}|} \\
T \Delta^{l(r-j, g-j)} \rightarrow 0, \quad j=0,1, \ldots .
\end{gathered}
$$


Тогда распределение случайной величины $\xi(D, F, B)$ сходится к распределению Пуассона с параметром $\lambda$.

Следствие 2. Пусть $K=G F(2), F(x)=A_{1} x+A_{2} f(x)+S(x)$, $\left\{f_{1}(x), \ldots, f_{m}(x)\right\} \supseteq \bar{Q}_{n}^{(g)}, \log _{2} n \leq g \leq n$, и выполнены условия $n, T \rightarrow \infty$,

$$
\begin{gathered}
2^{n-T}|B| \rightarrow \lambda, \quad 0<\lambda<\infty \\
|B| \leq 2^{\delta T}, \\
0 \leq \Delta \leq\left(2\left(1-n^{-1} \psi(n)\right)\right)^{1-\delta}-1,
\end{gathered}
$$

где $0 \leq \delta=\delta(n)=o(1), 0<\psi(n)=o(n) u$

$$
\frac{\ln ^{2} n}{\psi(n)}=o(1), n \rightarrow \infty
$$

Тогда распределение случайной величины $\xi\left(V^{n} \backslash\left\{0^{n}\right\}, F, B\right)$ сходится к распределению Пуассона с параметром $\lambda$.

ЗАМЕЧАНИЕ 4. При выполнении условий (6.7), (6.8) и (6.10) следствия 2 правая часть в (6.9) стремится к единице, что означает возможность асимптотического вырождения распределений (1.4) элементов матрицы $A=\left(A_{1}, A_{2}\right)$.

Немного о доказательстве теоремы 4. Вспомним, что при выводе теоремы 3 для проверки большинства условий теоремы Б. А. Севастьянова мы использовали схему и фрагменты доказательства теоремы 3 работы [2], добавив к ним новую выкладку для проверки условия (2.8). Для теоремы 4 все делается аналогично. Только теперь вместо теоремы 3 из [2] используется теорема 3 из [1]. Проверка же условия (2.8) здесь проводится точно так же, как для теоремы 3. Поэтому мы доказательство теоремы 4 не приводим.

Доказательство следствия 2 сводится к проверке условий $\rho\left(D_{f}\right) \rightarrow 0$, (6.3), (6.5) и (6.6) теоремы 4 в случае, когда $D=V^{n} \backslash\left\{0^{n}\right\}$. Проверка условий $\rho\left(D_{f}\right) \rightarrow 0$ и (6.3) повторяет проверку (5.5) в доказательстве следствия 1 (см. раздел 4). Проверка условий (6.5) и (6.6) приведена в доказательстве следствия 5 работы [1]. Поэтому эти выкладки мы опускаем.

Авторы благодарны А. М. Зубкову за полезные замечания. 


\section{Список литературы}

1. Копьтцев В. А., Михайлов В. Г. О распределении чисел решений случайных включений // Математические вопросы криптографии. - 2011. Т. 2. Вып. 2. - С. 55-80.

2. Копытиев B. А., Михайлов В. Г. Теоремы пуассоновского типа для числа решений случайных включений // Математические вопросы криптографии. - 2010. - Т. 1. Вып. 4. - С. 63-84.

3. Копытиев В. А., Михайлов В. Г. Теоремы пуассоновского типа для числа специальных решений случайного линейного включения // Дискретная математика. - 2010. - Т. 22. Вып. 2. - С. 3-21.

4. Михайлов В. Г. О предельной теореме Б. А. Севастьянова для сумм зависимых случайных индикаторов // Обозрение прикладной и промышленной математики. - 2003. - Т. 10. Вып. 3. - С. 571-578.

5. Колчин В. Ф., Севастьянов Б. А., Чистяков В. П. Случайные размещения. - М.: Наука, 1976. - 224 с.

6. Севастьянов Б. А. Предельный закон Пуассона в схеме сумм зависимых случайных величин // Теория вероятностей и ее применения. - 1972. Т. 17. Вып. 4. - С. 733-738.

7. Масол В. И. Теорема о предельном распределении числа ложных решений системы нелинейных случайных уравнений // Теория вероятностей и ее применения. - 1998. - Т. 43. Вып. 1. - С. 41-56. 